New Theory

ISSN: 2149-1402
35 (2021) 91-102

Journal of New Theory

https://dergipark.org.tr/en/pub/jnt

Open Access

\title{
Analysis and Simulation of a Two-Stage Blocked Tandem Queueing System
}

\author{
Erdinç Yücesoy ${ }^{1}$,, Vedat Sağlam ${ }^{2}$
}

\author{
Article History \\ Received: 17 May 2021 \\ Accepted: 08 Jun 2021 \\ Published: 30 Jun 2021 \\ Research Article
}

\begin{abstract}
In this paper, a new blocked tandem queueing model is given and analysed. The arrival process to this queueing model is Poisson with parameter $\lambda$. There is one service unit at the first stage of the system, and the service time of this unit is exponentially distributed with $\mu_{1}$ parameter. There are two parallel service units at the second stage, and the service time of these service units are exponentially distributed with parameters $\mu_{2}$ and $\mu_{3}$. No queue is allowed at the first stage of the system. Upon completing service at the first stage, a customer proceeds to the second stage if at least one of the service units at the second stage is available. If both service units at the second stage are busy, the customer blocks the service unit at the first stage, which results in loss. The most important measure of performance of this queueing system is the loss probability $\pi_{\text {loss }}$. First of all, the state probabilities of the system are obtained and then using these probabilities, the steady-state distribution of the system is obtained. Transition probabilities of the system are calculated by using steady-state probabilities, and finally an equation is obtained for $\pi_{\text {loss }}$ in terms of transition probabilities. Furthermore, another measure of performance, the mean number of customers, is obtained in terms of transition probabilities. Since the Equation for $\pi_{l o s s}$ is very complex, a numerical method is used to calculate the minimum $\pi_{\text {loss }}$ probabilities. After numerical optimal $\pi_{\text {loss }}$ calculations, a simulation of the queueing system is done, and it is seen that the obtained numerical $\pi_{\text {loss }}$ values tend to simulation results.
\end{abstract}

Keywords - Stochastic processes, Markov chains, queueing theory, optimization, queue performance

Mathematics Subject Classification (2020) - 60K25, 60K 30

\section{Introduction}

Stochastic queueing models are widely used in production lines, telecommunication technologies and computer sciences. In recent years, the studies mostly focused on queueing networks. A queueing network is simply a combination of several queueing systems. Bertsimas studied on performance analyse of queueing networks via Robust optimization [1]. A semi-open queueing network with a Markovian arrival process having a finite number of nodes is considered in a study [2]. A study on evaluating the performance of general queueing networks in manufacturing systems is given in [3]. Dudina et al. considered a multi-service retrial queueing system with Markovian arrival flow to model a call centre [4]. For the first time, Hunt [5] defined the customer's blocking effects in a queue sequence. Various performance measures, namely the average number of customers in the queueing system, the proportion of customers entering the queueing system, average waiting time, and a blocked series queue, have been obtained in the study [6]. Basharin et al. show

\footnotetext{
${ }^{1}$ erdincyucesoy@odu.edu.tr (Corresponding Author); ${ }^{2}$ vsaglam@omu.edu.tr ${ }^{1}$ Department of Mathematics, Faculty of Science, Ordu University, Ordu, Türkiye

${ }^{2}$ Department of Statistics, Faculty of Science, Ondokuz Mayıs University, Samsun, Türkiye
} 
how properties of Markovian Arrival Processes can be derived from the general theory of Markov processes with a homogeneous second component [7]. In another study [8], a two-station tandem queue with blocking is considered, and an accurate solution with correct stationary distribution is given. A stochastic queueing model, consisting of two heterogeneous service channels and having no waiting room, is considered [9]. In that study, Sağlam et al. calculated the expected number of customers and loss probability, an optimal ordering of service channels is given and minimizing parameters of the queueing system are found. In another related study [10], a queueing model with two sequential stations is constructed. In this model, there is a single service at each station, and no queue is allowed at the second station. The state probabilities and loss probability of this model are obtained. Furthermore, the model is simulated. In a recent study [2], a semi-open queueing network having a finite number of nodes is considered, and the stationary behaviour of queueing states is analysed.

In this paper, a new blocked tandem queueing system is constructed and analysed. The model we have analysed is a modified version of the model studied in [9]. The arrival process to this new queueing system is Poisson. There is one service unit at the first stage of the system, and the service time of this unit is exponentially distributed. There are two parallel service units at the second stage, and the service time of these service units are exponentially distributed. No queue is allowed at the first stage of the system. Upon completing service at the first stage, a customer proceeds to the second stage if at least one of the service units at the second stage is available. If both service units at the second stage are busy, the customer blocks the service unit at the first stage; hence, loss occurs. The most important measure of performance of this queueing system is the loss probability.

\section{The Stochastic Queueing Model}

The queueing model we considered in this study has Poisson arrival flow with parameter $\lambda$. At the first station, there is a single service unit that has exponentially distributed service time with parameter $\mu_{1}$ and no queue is allowed at this phase. The second station of the system consists of two parallel service units, and they also have exponentially distributed service times with parameters $\mu_{2}$ and $\mu_{3}$ respectively. As well as the first station, no queue is allowed at the second station. Upon receiving service at the first station, a customer proceeds to the second station of the queueing system. If both service units at the second station are empty, the customer enters the first service unit with probability $\alpha_{1}$ or second service unit with probability $\alpha_{2}=1-\alpha_{1}$. If only one of the service units at the second station is available, the customer proceeds to this server. On the other hand, if both servers at the second station are busy, the customer waits at the first station until any of the service units of the second station is available; hence the customer blocks the first station. This queueing model is mathematically stated as follows: At any given time $t$, let $\xi_{1}(t)$ random variable be the number of customers in service unit of the first station, $\xi_{2}(t)$ and $\xi_{3}(t)$ random variables be the number of customers in the services of the second station. Then the 3-dimensional continuous-time Markov chain of the model is stated as $\left\{\xi_{1}(t), \xi_{2}(t), \xi_{3}(t) ; t \geq 0\right\}$ and the state probabilities of the Markov chain is $p_{n_{1}, n_{2}, n_{3}}$ where $n_{1} \in\{0,1\}, n_{2} \in$ $\{0,1\}, n_{3} \in\{0,1\}$. Finally, the state space of the defined Markov chain is $\mathfrak{I}=$ $\{(0,0,0),(0,0,1),(0,1,0),(1,0,0),(0,1,1),(1,0,1),(1,1,0),(1,1,1),(b, 1,1)\}$.

\subsection{The Transition Probabilities of the Queueing System}

First, we need to find the state probabilities of the system to obtain transition probabilities. Then Kolmogorov differential equations are acquired using state probabilities, and by using Kolmogorov equations, the stationary distribution of the chain is obtained. Finally, the transition probabilities are found with the help of stationary distribution. The probability at any given time $t$, in which there are $n_{1}$ customers in the first service unit, $n_{2}$ customers in the second, and $n_{3}$ customers in the third, is defined as

$$
P\left(\xi_{1}(t)=n_{1}, \xi_{2}(t)=n_{2}, \xi_{3}(t)=n_{3}\right)=p_{n_{1} n_{2} n_{3}}(t)
$$

For $\Delta t \rightarrow 0$, the state probabilities of the Markov chain $\left\{\xi_{1}(t), \xi_{2}(t), \xi_{3}(t) ; t \geq 0\right\}$ are obtained as below: 


$$
\begin{aligned}
p_{000}(t+h)= & p_{000}(t)(1-\lambda h+o(h))+p_{010}(t)\left(\mu_{2} h+o(h)\right) \\
& +p_{001}(t)\left(\mu_{3} h+o(h)\right)+o(h) \\
p_{010}(t+h)= & p_{010}(t)(1-\lambda h+o(h))\left(1-\mu_{2} h+o(h)\right) \\
& +p_{011}(t)\left(\mu_{3} h+o(h)\right)+p_{100}(t) \alpha_{1}\left(\mu_{1} h+o(h)\right)+o(h) \\
p_{001}(t+h)= & p_{001}(t)(1-\lambda h+o(h))\left(1-\mu_{3} h+o(h)\right) \\
& +p_{011}(t)\left(\mu_{2} h+o(h)\right)+p_{100}(t) \alpha_{2}\left(\mu_{1} h+o(h)\right)+o(h) \\
p_{011}(t+h)= & p_{011}(t)(1-\lambda h+o(h))\left(1-\mu_{2} h+o(h)\right)\left(1-\mu_{3} h+o(h)\right) \\
& +p_{110}(t)\left(\mu_{1} h+o(h)\right)+p_{101}(t)\left(\mu_{1} h+o(h)\right)+p_{b 11}(t)\left(\mu_{2} h+o(h)\right) \\
& +p_{b 11}(t)\left(\mu_{3} h+o(h)\right)+o(h) \\
p_{100}(t+h)= & p_{100}(t)\left(1-\mu_{1} h+o(h)\right)+p_{000}(t)(\lambda h+o(h)) \\
& +p_{110}(t)\left(\mu_{2} h+o(h)\right)+p_{101}(t)\left(\mu_{3} h+o(h)\right)+o(h) \\
p_{110}(t+h)= & p_{110}(t)\left(1-\mu_{1} h+o(h)\right)\left(1-\mu_{2} h+o(h)\right) \\
& +p_{111}(t)\left(\mu_{3}+o(h)\right)+p_{010}(t)(\lambda h+o(h))+o(h) \\
& +p_{111}(t)\left(\mu_{1}+o(h)\right)+o(h) \\
p_{101}(t+h)= & p_{101}(t)\left(1-\mu_{1} h+o(h)\right)\left(1-\mu_{3} h+o(h)\right) \\
& +p_{111}(t)\left(\mu_{2}+o(h)\right)+p_{001}(t)(\lambda h+o(h))+o(h) \\
p_{b 11}(t+h)= & p_{b 11}(t)\left(1-\mu_{2} h+o(h)\right)\left(1-\mu_{3} h+o(h)\right) \\
p_{111}(t+h)= & p_{111}(t)\left(1-\mu_{1} h+o(h)\right)\left(1-\mu_{2} h+o(h)\right)\left(1-\mu_{3} h+o(h)\right) \\
& p_{011}(t)(\lambda h+o(h))+o(h) \\
&
\end{aligned}
$$

under the assumption of limit distribution by using state probabilities, we have the stationary state probabilities as following:

$$
\begin{gathered}
0=-\lambda p_{000}+\mu_{2} p_{010}+\mu_{3} p_{001} \\
0=-\left(\lambda+\mu_{2}\right) p_{010}+\mu_{3} p_{011}+\alpha_{1} \mu_{1} p_{100} \\
0=-\left(\lambda+\mu_{3}\right) p_{001}+\mu_{2} p_{011}+\alpha_{2} \mu_{1} p_{100} \\
0=-\left(\lambda+\mu_{2}+\mu_{3}\right) p_{011}+\mu_{1} p_{110}+\mu_{1} p_{101}+\mu_{2} p_{b 11}+\mu_{3} p_{b 11} \\
0=-\mu_{1} p_{100}+\lambda p_{000}+p_{110} \mu_{2}+p_{101} \mu_{3} \\
0=-\left(\mu_{1}+\mu_{2}\right) p_{110}+\mu_{3} p_{111}+\lambda p_{010} \\
0=-\left(\mu_{1}+\mu_{3}\right) p_{101}+\mu_{2} p_{111}+\lambda p_{001} \\
0=-\left(\mu_{1}+\mu_{2}+\mu_{3}\right) p_{111}+\lambda p_{011} \\
0=-\left(\mu_{2}+\mu_{3}\right) p_{b 11}+\mu_{1} p_{111}
\end{gathered}
$$

Now, we calculate the transition probabilities of the queueing system by using stationary state probabilities. Using Equation (2.17), we have 


$$
p_{111}=\left(\frac{\lambda}{\mu_{1}+\mu_{2}+\mu_{3}}\right) p_{011}
$$

Equations (2.18) and (2.19) lead us to:

$$
p_{b 11}=\left(\frac{\mu_{1}}{\mu_{2}+\mu_{3}}\right)\left(\frac{\lambda}{\mu_{1}+\mu_{2}+\mu_{3}}\right) p_{011}
$$

With the same solution manner, all transition probabilities are obtained in terms of $p_{011}$ :

$$
\begin{gathered}
p_{001}=\left[\frac{\mu_{2}}{\lambda+\mu_{3}}+\alpha_{2} \frac{\mu_{1}}{\lambda+\mu_{3}} \Delta_{4}\right] p_{011} \\
p_{010}=\left[\frac{\mu_{3}}{\lambda+\mu_{2}}+\alpha_{1} \frac{\mu_{1}}{\lambda+\mu_{2}} \Delta_{4}\right] p_{011} \\
p_{000}=\left[\frac{\mu_{2}}{\lambda}\left(\frac{\mu_{3}}{\lambda+\mu_{2}}+\alpha_{1} \frac{\mu_{1}}{\lambda+\mu_{2}} \Delta_{4}\right)+\frac{\mu_{3}}{\lambda}\left(\frac{\mu_{2}}{\lambda+\mu_{3}}+\alpha_{2} \frac{\mu_{1}}{\lambda+\mu_{3}} \Delta_{4}\right)\right] p_{011} \\
p_{101}=\left[\frac{\mu_{2}}{\mu_{1}+\mu_{3}} \cdot \frac{\lambda}{\mu_{1}+\mu_{2}+\mu_{3}}+\frac{\lambda}{\mu_{1}+\mu_{3}} \cdot \frac{\mu_{2}}{\lambda+\mu_{3}}+\alpha_{2} \frac{\lambda}{\mu_{1}+\mu_{3}} \cdot \frac{\mu_{1}}{\lambda+\mu_{3}} \Delta_{4}\right] p_{011} \\
p_{100}=\Delta_{4} \cdot p_{011} \\
\left.\lambda \mu_{3}=\left[\frac{\lambda}{\left(\mu_{1}+\mu_{2}\right)\left(\mu_{1}+\mu_{2}+\mu_{3}\right)}+\frac{\mu_{3}}{\mu_{1}+\mu_{2}}+\alpha_{1} \frac{\mu_{1}}{\lambda+\mu_{2}} \Delta_{4}\right)\right] p_{011}
\end{gathered}
$$

where,

$$
\begin{aligned}
& \Delta_{1}=1-\alpha_{1} \frac{\mu_{2}}{\lambda+\mu_{2}}\left(1+\frac{\lambda}{\mu_{1}+\mu_{2}}\right)-\alpha_{2} \frac{\mu_{3}}{\lambda+\mu_{3}}\left(1+\frac{\lambda}{\mu_{1}+\mu_{3}}\right) \\
& \Delta_{2}=\frac{\mu_{3}}{\lambda+\mu_{2}}+\left(\frac{\mu_{3}}{\mu_{1}+\mu_{2}} \cdot \frac{\lambda}{\mu_{1}+\mu_{2}+\mu_{3}}\right)+\left(\frac{\lambda}{\mu_{1}+\mu_{2}} \cdot \frac{\mu_{3}}{\lambda+\mu_{2}}\right) \\
& \Delta_{3}=\frac{\mu_{2}}{\lambda+\mu_{3}}+\left(\frac{\mu_{2}}{\mu_{1}+\mu_{3}} \cdot \frac{\lambda}{\mu_{1}+\mu_{2}+\mu_{3}}\right)+\left(\frac{\lambda}{\mu_{1}+\mu_{3}} \cdot \frac{\mu_{2}}{\lambda+\mu_{3}}\right)
\end{aligned}
$$

and

$$
\Delta_{4}=\frac{\mu_{2} \Delta_{2}+\mu_{3} \Delta_{3}}{\mu_{1} \Delta_{1}}
$$

Hence, the sum of all these probabilities is 1, i.e.,

$$
p_{000}+p_{001}+p_{010}+p_{100}+p_{011}+p_{101}+p_{110}+p_{111}+p_{b 11}=1
$$

Substituting all obtained transition probabilities in Equation (2.27), we have

$$
\begin{aligned}
& p_{011}\left[\frac{\mu_{2}}{\lambda}\left(\frac{\mu_{3}}{\lambda+\mu_{2}}+\alpha_{1} \frac{\mu_{1}}{\lambda+\mu_{2}} \Delta_{4}\right)+\frac{\mu_{3}}{\lambda}\left(\frac{\mu_{2}}{\lambda+\mu_{3}}+\alpha_{2} \frac{\mu_{1}}{\lambda+\mu_{3}} \Delta_{4}\right)\right. \\
& +\left(\frac{\mu_{2}}{\lambda+\mu_{3}}+\alpha_{2} \frac{\mu_{1}}{\lambda+\mu_{3}} \Delta_{4}\right)+\left(\frac{\mu_{3}}{\lambda+\mu_{2}}+\alpha_{1} \frac{\mu_{1}}{\lambda+\mu_{2}} \Delta_{4}\right)+\Delta_{4}+1 \\
& +\left(\frac{\mu_{2}}{\mu_{1}+\mu_{3}} \cdot \frac{\lambda}{\mu_{1}+\mu_{2}+\mu_{3}}+\frac{\lambda}{\mu_{1}+\mu_{3}} \cdot \frac{\mu_{2}}{\lambda+\mu_{3}}+\alpha_{2} \frac{\lambda}{\mu_{1}+\mu_{3}} \cdot \frac{\mu_{1}}{\lambda+\mu_{3}} \Delta_{4}\right)
\end{aligned}
$$




$$
\begin{aligned}
& +\left(\frac{\lambda \mu_{3}}{\left(\mu_{1}+\mu_{2}\right)\left(\mu_{1}+\mu_{2}+\mu_{3}\right)}+\frac{\lambda}{\mu_{1}+\mu_{2}}\left(\frac{\mu_{3}}{\lambda+\mu_{2}}+\alpha_{1} \frac{\mu_{1}}{\lambda+\mu_{2}} \Delta_{4}\right)\right) \\
& \left.+\left(\frac{\lambda}{\mu_{1}+\mu_{2}+\mu_{3}}\right)+\left(\frac{\mu_{1}}{\mu_{2}+\mu_{3}}\right)\left(\frac{\lambda}{\mu_{1}+\mu_{2}+\mu_{3}}\right)\right] \\
& =1
\end{aligned}
$$

Note that the last Equation is only in terms of $p_{011}$. Hereby, the transition probability $p_{011}$ is obtained in terms of system parameters precisely as:

$$
\begin{aligned}
p_{011} & =\left[\frac{\mu_{2}}{\lambda}\left(\frac{\mu_{3}}{\lambda+\mu_{2}}+\alpha_{1} \frac{\mu_{1}}{\lambda+\mu_{2}} \Delta_{4}\right)+\frac{\mu_{3}}{\lambda}\left(\frac{\mu_{2}}{\lambda+\mu_{3}}+\alpha_{2} \frac{\mu_{1}}{\lambda+\mu_{3}} \Delta_{4}\right)\right. \\
& +\left(\frac{\mu_{2}}{\lambda+\mu_{3}}+\alpha_{2} \frac{\mu_{1}}{\lambda+\mu_{3}} \Delta_{4}\right)+\left(\frac{\mu_{3}}{\lambda+\mu_{2}}+\alpha_{1} \frac{\mu_{1}}{\lambda+\mu_{2}} \Delta_{4}\right)+\Delta_{4}+1 \\
& +\left(\frac{\mu_{2}}{\mu_{1}+\mu_{3}} \cdot \frac{\lambda}{\mu_{1}+\mu_{2}+\mu_{3}}+\frac{\lambda}{\mu_{1}+\mu_{3}} \cdot \frac{\mu_{2}}{\lambda+\mu_{3}}+\alpha_{2} \frac{\lambda}{\mu_{1}+\mu_{3}} \cdot \frac{\mu_{1}}{\lambda+\mu_{3}} \Delta_{4}\right) \\
& +\left(\frac{\lambda \mu_{3}}{\left(\mu_{1}+\mu_{2}\right)\left(\mu_{1}+\mu_{2}+\mu_{3}\right)}+\frac{\lambda}{\mu_{1}+\mu_{2}}\left(\frac{\mu_{3}}{\lambda+\mu_{2}}+\alpha_{1} \frac{\mu_{1}}{\lambda+\mu_{2}} \Delta_{4}\right)\right) \\
& \left.+\left(\frac{\lambda}{\mu_{1}+\mu_{2}+\mu_{3}}\right)+\left(\frac{\mu_{1}}{\mu_{2}+\mu_{3}}\right)\left(\frac{\lambda}{\mu_{1}+\mu_{2}+\mu_{3}}\right)\right]^{-1}
\end{aligned}
$$

The last thing to do is to rewrite all transition probabilities in terms of system parameters:

$$
\begin{aligned}
p_{001} & =\left[\frac{\mu_{2}}{\lambda+\mu_{3}}+\alpha_{2} \frac{\mu_{1}}{\lambda+\mu_{3}} \Delta_{4}\right] \cdot\left[\frac{\mu_{2}}{\lambda}\left(\frac{\mu_{3}}{\lambda+\mu_{2}}+\alpha_{1} \frac{\mu_{1}}{\lambda+\mu_{2}} \Delta_{4}\right)+\frac{\mu_{3}}{\lambda}\left(\frac{\mu_{2}}{\lambda+\mu_{3}}+\alpha_{2} \frac{\mu_{1}}{\lambda+\mu_{3}} \Delta_{4}\right)\right. \\
& +\left(\frac{\mu_{2}}{\lambda+\mu_{3}}+\alpha_{2} \frac{\mu_{1}}{\lambda+\mu_{3}} \Delta_{4}\right)+\left(\frac{\mu_{3}}{\lambda+\mu_{2}}+\alpha_{1} \frac{\mu_{1}}{\lambda+\mu_{2}} \Delta_{4}\right)+\Delta_{4}+1 \\
& +\left(\frac{\mu_{2}}{\mu_{1}+\mu_{3}} \cdot \frac{\lambda}{\mu_{1}+\mu_{2}+\mu_{3}}+\frac{\lambda}{\mu_{1}+\mu_{3}} \cdot \frac{\mu_{2}}{\lambda+\mu_{3}}+\alpha_{2} \frac{\lambda}{\mu_{1}+\mu_{3}} \cdot \frac{\mu_{1}}{\lambda+\mu_{3}} \Delta_{4}\right) \\
& +\left(\frac{\lambda \mu_{3}}{\left(\mu_{1}+\mu_{2}\right)\left(\mu_{1}+\mu_{2}+\mu_{3}\right)}+\frac{\lambda}{\mu_{1}+\mu_{2}}\left(\frac{\mu_{3}}{\lambda+\mu_{2}}+\alpha_{1} \frac{\mu_{1}}{\lambda+\mu_{2}} \Delta_{4}\right)\right) \\
& \left.+\left(\frac{\lambda}{\mu_{1}+\mu_{2}+\mu_{3}}\right)+\left(\frac{\mu_{1}}{\mu_{2}+\mu_{3}}\right)\left(\frac{\lambda}{\mu_{1}+\mu_{2}+\mu_{3}}\right)\right] \\
= & {\left[\frac{\mu_{3}}{\lambda+\mu_{2}}+\alpha_{1} \frac{\mu_{1}}{\lambda+\mu_{2}} \Delta_{4}\right] \cdot\left[\frac{\mu_{2}}{\lambda}\left(\frac{\mu_{3}}{\lambda+\mu_{2}}+\alpha_{1} \frac{\mu_{1}}{\lambda+\mu_{2}} \Delta_{4}\right)+\frac{\mu_{3}}{\lambda}\left(\frac{\mu_{2}}{\lambda+\mu_{3}}+\alpha_{2} \frac{\mu_{1}}{\lambda+\mu_{3}} \Delta_{4}\right)\right.} \\
& +\left(\frac{\mu_{2}}{\lambda+\mu_{3}}+\alpha_{2} \frac{\mu_{1}}{\lambda+\mu_{3}} \Delta_{4}\right)+\left(\frac{\mu_{3}}{\lambda+\mu_{2}}+\alpha_{1} \frac{\mu_{1}}{\lambda+\mu_{2}} \Delta_{4}\right)+\Delta_{4}+1
\end{aligned}
$$




$$
\begin{aligned}
& +\left(\frac{\mu_{2}}{\mu_{1}+\mu_{3}} \cdot \frac{\lambda}{\mu_{1}+\mu_{2}+\mu_{3}}+\frac{\lambda}{\mu_{1}+\mu_{3}} \cdot \frac{\mu_{2}}{\lambda+\mu_{3}}+\alpha_{2} \frac{\lambda}{\mu_{1}+\mu_{3}} \cdot \frac{\mu_{1}}{\lambda+\mu_{3}} \Delta_{4}\right) \\
& +\left(\frac{\lambda \mu_{3}}{\left(\mu_{1}+\mu_{2}\right)\left(\mu_{1}+\mu_{2}+\mu_{3}\right)}+\frac{\lambda}{\mu_{1}+\mu_{2}}\left(\frac{\mu_{3}}{\lambda+\mu_{2}}+\alpha_{1} \frac{\mu_{1}}{\lambda+\mu_{2}} \Delta_{4}\right)\right) \\
& \left.+\left(\frac{\lambda}{\mu_{1}+\mu_{2}+\mu_{3}}\right)+\left(\frac{\mu_{1}}{\mu_{2}+\mu_{3}}\right)\left(\frac{\lambda}{\mu_{1}+\mu_{2}+\mu_{3}}\right)\right]^{-1} \\
& p_{000}=\left[\frac{\mu_{2}}{\lambda}\left(\frac{\mu_{3}}{\lambda+\mu_{2}}+\alpha_{1} \frac{\mu_{1}}{\lambda+\mu_{2}} \Delta_{4}\right)+\frac{\mu_{3}}{\lambda}\left(\frac{\mu_{2}}{\lambda+\mu_{3}}+\alpha_{2} \frac{\mu_{1}}{\lambda+\mu_{3}} \Delta_{4}\right)\right] \\
& \cdot\left[\frac{\mu_{2}}{\lambda}\left(\frac{\mu_{3}}{\lambda+\mu_{2}}+\alpha_{1} \frac{\mu_{1}}{\lambda+\mu_{2}} \Delta_{4}\right)+\frac{\mu_{3}}{\lambda}\left(\frac{\mu_{2}}{\lambda+\mu_{3}}+\alpha_{2} \frac{\mu_{1}}{\lambda+\mu_{3}} \Delta_{4}\right)\right. \\
& +\left(\frac{\mu_{2}}{\lambda+\mu_{3}}+\alpha_{2} \frac{\mu_{1}}{\lambda+\mu_{3}} \Delta_{4}\right)+\left(\frac{\mu_{3}}{\lambda+\mu_{2}}+\alpha_{1} \frac{\mu_{1}}{\lambda+\mu_{2}} \Delta_{4}\right)+\Delta_{4}+1 \\
& +\left(\frac{\mu_{2}}{\mu_{1}+\mu_{3}} \cdot \frac{\lambda}{\mu_{1}+\mu_{2}+\mu_{3}}+\frac{\lambda}{\mu_{1}+\mu_{3}} \cdot \frac{\mu_{2}}{\lambda+\mu_{3}}+\alpha_{2} \frac{\lambda}{\mu_{1}+\mu_{3}} \cdot \frac{\mu_{1}}{\lambda+\mu_{3}} \Delta_{4}\right) \\
& +\left(\frac{\lambda \mu_{3}}{\left(\mu_{1}+\mu_{2}\right)\left(\mu_{1}+\mu_{2}+\mu_{3}\right)}+\frac{\lambda}{\mu_{1}+\mu_{2}}\left(\frac{\mu_{3}}{\lambda+\mu_{2}}+\alpha_{1} \frac{\mu_{1}}{\lambda+\mu_{2}} \Delta_{4}\right)\right) \\
& \left.+\left(\frac{\lambda}{\mu_{1}+\mu_{2}+\mu_{3}}\right)+\left(\frac{\mu_{1}}{\mu_{2}+\mu_{3}}\right)\left(\frac{\lambda}{\mu_{1}+\mu_{2}+\mu_{3}}\right)\right]^{-1} \\
& p_{101}=\left(\frac{\mu_{2}}{\mu_{1}+\mu_{3}} \cdot \frac{\lambda}{\mu_{1}+\mu_{2}+\mu_{3}}+\frac{\lambda}{\mu_{1}+\mu_{3}} \cdot \frac{\mu_{2}}{\lambda+\mu_{3}}+\alpha_{2} \frac{\lambda}{\mu_{1}+\mu_{3}} \cdot \frac{\mu_{1}}{\lambda+\mu_{3}} \Delta_{4}\right) \\
& \cdot\left[\frac{\mu_{2}}{\lambda}\left(\frac{\mu_{3}}{\lambda+\mu_{2}}+\alpha_{1} \frac{\mu_{1}}{\lambda+\mu_{2}} \Delta_{4}\right)+\frac{\mu_{3}}{\lambda}\left(\frac{\mu_{2}}{\lambda+\mu_{3}}+\alpha_{2} \frac{\mu_{1}}{\lambda+\mu_{3}} \Delta_{4}\right)\right. \\
& +\left(\frac{\mu_{2}}{\lambda+\mu_{3}}+\alpha_{2} \frac{\mu_{1}}{\lambda+\mu_{3}} \Delta_{4}\right)+\left(\frac{\mu_{3}}{\lambda+\mu_{2}}+\alpha_{1} \frac{\mu_{1}}{\lambda+\mu_{2}} \Delta_{4}\right)+\Delta_{4}+1 \\
& +\left(\frac{\mu_{2}}{\mu_{1}+\mu_{3}} \cdot \frac{\lambda}{\mu_{1}+\mu_{2}+\mu_{3}}+\frac{\lambda}{\mu_{1}+\mu_{3}} \cdot \frac{\mu_{2}}{\lambda+\mu_{3}}+\alpha_{2} \frac{\lambda}{\mu_{1}+\mu_{3}} \cdot \frac{\mu_{1}}{\lambda+\mu_{3}} \Delta_{4}\right) \\
& +\left(\frac{\lambda \mu_{3}}{\left(\mu_{1}+\mu_{2}\right)\left(\mu_{1}+\mu_{2}+\mu_{3}\right)}+\frac{\lambda}{\mu_{1}+\mu_{2}}\left(\frac{\mu_{3}}{\lambda+\mu_{2}}+\alpha_{1} \frac{\mu_{1}}{\lambda+\mu_{2}} \Delta_{4}\right)\right) \\
& \left.+\left(\frac{\lambda}{\mu_{1}+\mu_{2}+\mu_{3}}\right)+\left(\frac{\mu_{1}}{\mu_{2}+\mu_{3}}\right)\left(\frac{\lambda}{\mu_{1}+\mu_{2}+\mu_{3}}\right)\right]^{-1}
\end{aligned}
$$




$$
\begin{aligned}
& p_{100}=\Delta_{4} \cdot\left[\frac{\mu_{2}}{\lambda}\left(\frac{\mu_{3}}{\lambda+\mu_{2}}+\alpha_{1} \frac{\mu_{1}}{\lambda+\mu_{2}} \Delta_{4}\right)+\frac{\mu_{3}}{\lambda}\left(\frac{\mu_{2}}{\lambda+\mu_{3}}+\alpha_{2} \frac{\mu_{1}}{\lambda+\mu_{3}} \Delta_{4}\right)\right. \\
& +\left(\frac{\mu_{2}}{\lambda+\mu_{3}}+\alpha_{2} \frac{\mu_{1}}{\lambda+\mu_{3}} \Delta_{4}\right)+\left(\frac{\mu_{3}}{\lambda+\mu_{2}}+\alpha_{1} \frac{\mu_{1}}{\lambda+\mu_{2}} \Delta_{4}\right)+\Delta_{4}+1 \\
& +\left(\frac{\mu_{2}}{\mu_{1}+\mu_{3}} \cdot \frac{\lambda}{\mu_{1}+\mu_{2}+\mu_{3}}+\frac{\lambda}{\mu_{1}+\mu_{3}} \cdot \frac{\mu_{2}}{\lambda+\mu_{3}}+\alpha_{2} \frac{\lambda}{\mu_{1}+\mu_{3}} \cdot \frac{\mu_{1}}{\lambda+\mu_{3}} \Delta_{4}\right) \\
& +\left(\frac{\lambda \mu_{3}}{\left(\mu_{1}+\mu_{2}\right)\left(\mu_{1}+\mu_{2}+\mu_{3}\right)}+\frac{\lambda}{\mu_{1}+\mu_{2}}\left(\frac{\mu_{3}}{\lambda+\mu_{2}}+\alpha_{1} \frac{\mu_{1}}{\lambda+\mu_{2}} \Delta_{4}\right)\right) \\
& \left.+\left(\frac{\lambda}{\mu_{1}+\mu_{2}+\mu_{3}}\right)+\left(\frac{\mu_{1}}{\mu_{2}+\mu_{3}}\right)\left(\frac{\lambda}{\mu_{1}+\mu_{2}+\mu_{3}}\right)\right]^{-1} \\
& p_{110}=\left[\frac{\lambda \mu_{3}}{\left(\mu_{1}+\mu_{2}\right)\left(\mu_{1}+\mu_{2}+\mu_{3}\right)}+\frac{\lambda}{\mu_{1}+\mu_{2}}\left(\frac{\mu_{3}}{\lambda+\mu_{2}}+\alpha_{1} \frac{\mu_{1}}{\lambda+\mu_{2}} \Delta_{4}\right)\right] \\
& \cdot\left[\frac{\mu_{2}}{\lambda}\left(\frac{\mu_{3}}{\lambda+\mu_{2}}+\alpha_{1} \frac{\mu_{1}}{\lambda+\mu_{2}} \Delta_{4}\right)+\frac{\mu_{3}}{\lambda}\left(\frac{\mu_{2}}{\lambda+\mu_{3}}+\alpha_{2} \frac{\mu_{1}}{\lambda+\mu_{3}} \Delta_{4}\right)\right. \\
& +\left(\frac{\mu_{2}}{\lambda+\mu_{3}}+\alpha_{2} \frac{\mu_{1}}{\lambda+\mu_{3}} \Delta_{4}\right)+\left(\frac{\mu_{3}}{\lambda+\mu_{2}}+\alpha_{1} \frac{\mu_{1}}{\lambda+\mu_{2}} \Delta_{4}\right)+\Delta_{4}+1 \\
& +\left(\frac{\mu_{2}}{\mu_{1}+\mu_{3}} \cdot \frac{\lambda}{\mu_{1}+\mu_{2}+\mu_{3}}+\frac{\lambda}{\mu_{1}+\mu_{3}} \cdot \frac{\mu_{2}}{\lambda+\mu_{3}}+\alpha_{2} \frac{\lambda}{\mu_{1}+\mu_{3}} \cdot \frac{\mu_{1}}{\lambda+\mu_{3}} \Delta_{4}\right) \\
& +\left(\frac{\lambda \mu_{3}}{\left(\mu_{1}+\mu_{2}\right)\left(\mu_{1}+\mu_{2}+\mu_{3}\right)}+\frac{\lambda}{\mu_{1}+\mu_{2}}\left(\frac{\mu_{3}}{\lambda+\mu_{2}}+\alpha_{1} \frac{\mu_{1}}{\lambda+\mu_{2}} \Delta_{4}\right)\right) \\
& \left.+\left(\frac{\lambda}{\mu_{1}+\mu_{2}+\mu_{3}}\right)+\left(\frac{\mu_{1}}{\mu_{2}+\mu_{3}}\right)\left(\frac{\lambda}{\mu_{1}+\mu_{2}+\mu_{3}}\right)\right]^{-1}
\end{aligned}
$$

\subsection{The Loss Probability and The Mean Customer Number of the Queue}

The most important performance measure of the defined queueing model is the loss probability. In this queueing model, customer loss occurs only at the first station of the queue, as previously stated. In this context, the loss probability can be simply calculated as:

$$
\pi_{\text {loss }}=p_{110}+p_{100}+p_{101}+p_{b 11}+p_{111}
$$

Substituting the previously calculated probabilities $p_{110}, p_{100}, p_{101}, p_{b 11}$, and $p_{111}$, which are the equations (2.26), (2.25), (2.24), (2.20), and (2.19), respectively, in Equation (2.28); we precisely have loss probability in terms of system parameters as below:

$$
\pi_{\text {loss }}=\left[\frac{\lambda}{\mu_{1}+\mu_{2}+\mu_{3}}+\left(\frac{\mu_{1}}{\mu_{2}+\mu_{3}}\right)\left(\frac{\lambda}{\mu_{1}+\mu_{2}+\mu_{3}}\right)\right.
$$




$$
\begin{aligned}
& +\left(\frac{\mu_{2}}{\mu_{1}+\mu_{3}} \cdot \frac{\lambda}{\mu_{1}+\mu_{2}+\mu_{3}}+\frac{\lambda}{\mu_{1}+\mu_{3}} \cdot \frac{\mu_{2}}{\lambda+\mu_{3}}+\alpha_{2} \frac{\lambda}{\mu_{1}+\mu_{3}} \cdot \frac{\mu_{1}}{\lambda+\mu_{3}} \Delta_{4}\right)+\Delta_{4} \\
& \left.+\left(\frac{\lambda \mu_{3}}{\left(\mu_{1}+\mu_{2}\right)\left(\mu_{1}+\mu_{2}+\mu_{3}\right)}+\frac{\lambda}{\mu_{1}+\mu_{2}}\left(\frac{\mu_{3}}{\lambda+\mu_{2}}+\alpha_{1} \frac{\mu_{1}}{\lambda+\mu_{2}} \Delta_{4}\right)\right)\right] p_{011}
\end{aligned}
$$

Furthermore, the mean number of customers in the system is obtained as:

$$
E(N)=\sum_{n_{1} \in \mathfrak{J}} \sum_{n_{2} \in \mathfrak{I}} \sum_{n_{3} \in \mathfrak{I}}\left(n_{1}+n_{2}+n_{3}\right) p_{n_{1}, n_{2}, n_{3}}
$$

where $\mathfrak{I}=\{(0,0,0),(0,0,1),(0,1,0),(1,0,0),(0,1,1),(1,0,1),(1,1,0),(1,1,1),(b, 1,1)\}$ is the state space of the defined Markov chain. Hence the mean number of customers in the system is calculated as follows:

$$
E(N)=0 . p_{000}+1 .\left(p_{100}+p_{010}+p_{001}\right)+2 .\left(p_{110}+p_{101}+p_{011}\right)+3 \cdot\left(p_{111}+p_{b 11}\right)
$$

\subsection{The Optimization of the Loss Probability}

In a blocked queueing system, one of the most notable performance measures is the loss probability. When a service is busy, a new incoming customer cannot enter the service and has two options: to leave the system without having service or wait until the service is available. In the system we are interested in, when a service is busy, the incoming customer leaves the queue system without being served, so that loss occurs. The probability of this loss is called the loss probability. In this section, we aim to minimize the loss probability $\pi_{\text {loss }}$, obtained in the previous section, and calculate the mean customer number in the queueing system. When the loss probability, given with the Equation (2.29), is examined; clearly, it is very complex and difficult to reach the minimum value of $\pi_{\text {loss }}$ with the help of algebraic methods. Therefore, under two configurations of the queuing system, the loss probability is numerically calculated, and the minimum loss probability values of both configurations are determined. These two different configurations of the queuing system are based on customer arrival rate and the total service capacity of the system. In order to calculate the loss probability and mean number of customers, the arrival rate $\lambda$ is chosen as constant. In this context, $c=2 \lambda$ and $c=\lambda$ configurations are established where $c=\mu_{1}+\mu_{2}+\mu_{3}$ is the total service capacity of the queueing system.

First, the optimal service capacity of the $1 s t$ service unit is searched, and in the next step, the optimal service capacities of the $2^{\text {nd }}$ and $3^{\text {rd }}$ service units are determined for the changing $\alpha_{1}$ and $\alpha_{2}$ possibilities. The data obtained by applying this method are given in Table 1, 2, 3, and 4. According to the data obtained from the tables, minimum $\pi_{\text {loss }}$ values for $c=2 \lambda$ and $c=\lambda$ configurations are found. The results are also shown in Figure 1 and 2.

Table 1. Optimal service capacity selection of $1^{\text {st }}$ service unit for $c=2 \lambda$ system configuration and the corresponding loss probability and mean customer number values

\begin{tabular}{|c|c|c|c|c|c|c|c|}
\hline$\lambda$ & $\mu_{1}$ & $\mu_{2}$ & $\mu_{3}$ & $\alpha_{1}$ & $\alpha_{2}$ & $\pi_{\text {loss }}$ & $E(N)$ \\
\hline 2 & 0.2 & 1.9 & 1.9 & 0.5 & 0.5 & 0.9090 & 1.0047 \\
\hline 2 & 0.8 & 1.6 & 1.6 & 0.5 & 0.5 & 0.7150 & 1.0712 \\
\hline 2 & 1.4 & 1.3 & 1.3 & 0.5 & 0.5 & 0.5955 & 1.2177 \\
\hline 2 & 2 & 1 & 1 & 0.5 & 0.5 & 0.5322 & 1.4677 \\
\hline 2 & 2.2424 & 0.8889 & 0.8889 & 0.5 & 0.5 & 0.5233 & 1.5958 \\
\hline 2 & 3 & 0.5 & 0.5 & 0.5 & 0.5 & 0.6013 & 2.1958 \\
\hline 2 & 3.6 & 0.2 & 0.2 & 0.5 & 0.5 & 0.8073 & 2.7342 \\
\hline
\end{tabular}


Table 2. Selection of optimal service capacities of $2 n d$ and $3 r d$ service units for $c=2 \lambda$ system configuration and the corresponding loss probability and mean customer number values

\begin{tabular}{|c|c|c|c|c|c|c|c|}
\hline$\lambda$ & $\mu_{1}$ & $\mu_{2}$ & $\mu_{3}$ & $\alpha_{1}$ & $\alpha_{2}$ & $\pi_{\text {loss }}$ & $E(N)$ \\
\hline 2 & 2.2424 & 0.2 & 1.5575 & 0.5 & 0.5 & 0.5389 & 1.8454 \\
\hline 2 & 2.2424 & 0.8889 & 0.8889 & 0.5 & 0.5 & 0.5233 & 1.5958 \\
\hline 2 & 2.2424 & 1.7 & 0.0575 & 0.5 & 0.5 & 0.5474 & 1.9877 \\
\hline
\end{tabular}

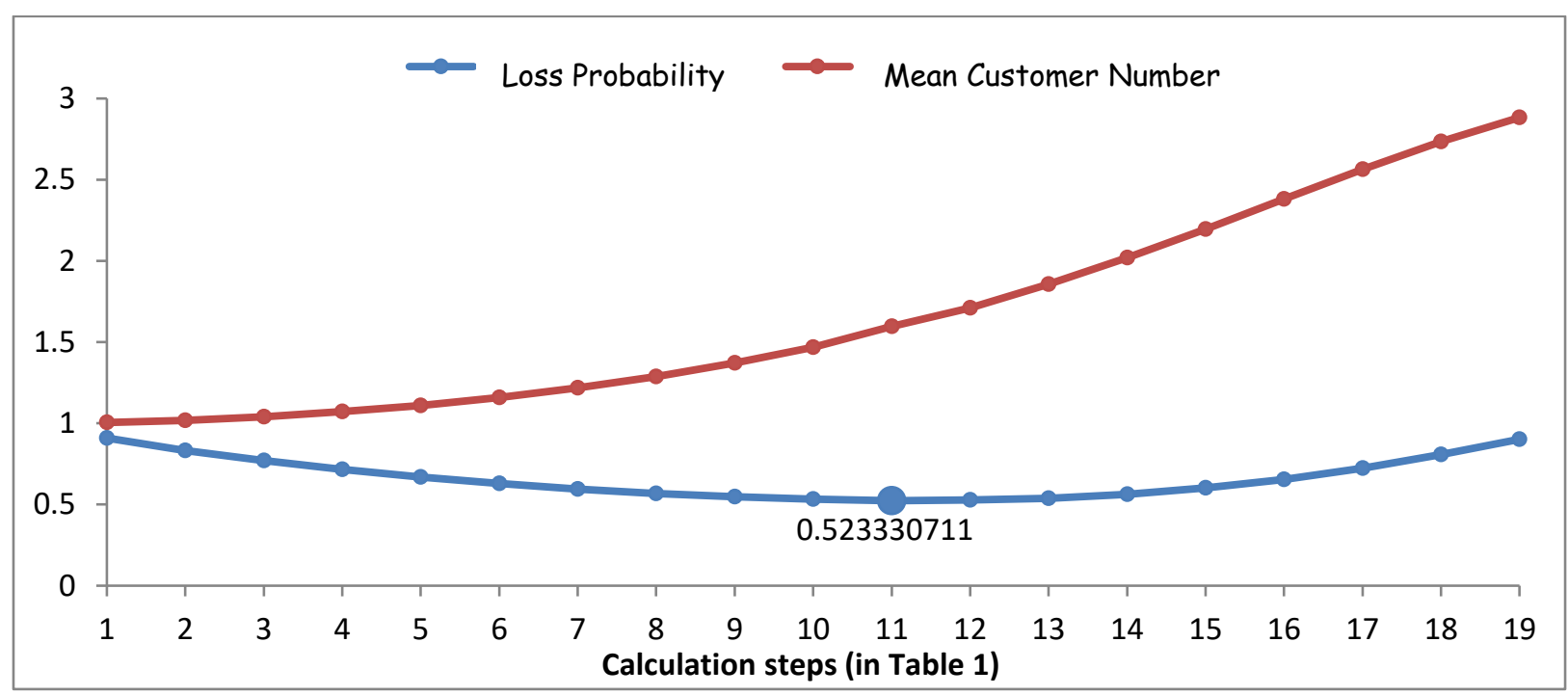

Fig. 1. The obtained $\pi_{\text {loss }}$ and $E(N)$ values for $c=2 \lambda$ configuration

As seen in Figure 1 , under the condition $\mu_{1}=\mu_{2}$ and $\alpha_{1}=\alpha_{2}$, minimum $\pi_{\text {loss }}=0.523330711$ is obtained for $c=2 \lambda$ configuration.

Table 3. Optimal service capacity selection of $1^{\text {st }}$ service unit for $c=\lambda$ system configuration and the corresponding loss probability and mean customer number values

\begin{tabular}{|c|c|c|c|c|c|c|c|}
\hline$\lambda$ & $\mu_{1}$ & $\mu_{2}$ & $\mu_{3}$ & $\alpha_{1}$ & $\alpha_{2}$ & $\pi_{\text {loss }}$ & $E(N)$ \\
\hline 2 & 0.2 & 0.9 & 0.9 & 0.5 & 0.5 & 0.9091 & 1.1110 \\
\hline 2 & 0.8 & 0.6 & 0.6 & 0.5 & 0.5 & 0.7316 & 1.6262 \\
\hline 2 & 1.07 & 0.465 & 0.465 & 0.5 & 0.5 & 0.7079 & 1.9639 \\
\hline 2 & 1.5 & 0.25 & 0.25 & 0.5 & 0.5 & 0.7785 & 2.5503 \\
\hline 2 & 1.9 & 0.05 & 0.05 & 0.5 & 0.5 & 0.9501 & 2.9422 \\
\hline
\end{tabular}


Table 4. Selection of optimal service capacities of $2^{\text {nd }}$ and $3^{\text {rd }}$ service units for $c=\lambda$ system configuration and the corresponding loss probability and mean customer number values

\begin{tabular}{|c|c|c|c|c|c|c|c|}
\hline$\lambda$ & $\mu_{1}$ & $\mu_{2}$ & $\mu_{3}$ & $\alpha_{1}$ & $\alpha_{2}$ & $\pi_{\text {loss }}$ & $E(N)$ \\
\hline 2 & 1.07 & 0.1 & 0.83 & 0.5 & 0.5 & 0.7183 & 2.1628 \\
\hline 2 & 1.07 & 0.4 & 0.53 & 0.5 & 0.5 & 0.7082 & 1.9695 \\
\hline 2 & 1.07 & 0.465 & 0.465 & 0.5 & 0.5 & 0.7079 & 1.9639 \\
\hline 2 & 1.07 & 0.7 & 0.23 & 0.5 & 0.5 & 0.7120 & 2.0406 \\
\hline 2 & 1.07 & 0.9 & 0.03 & 0.5 & 0.5 & 0.7236 & 2.2625 \\
\hline
\end{tabular}

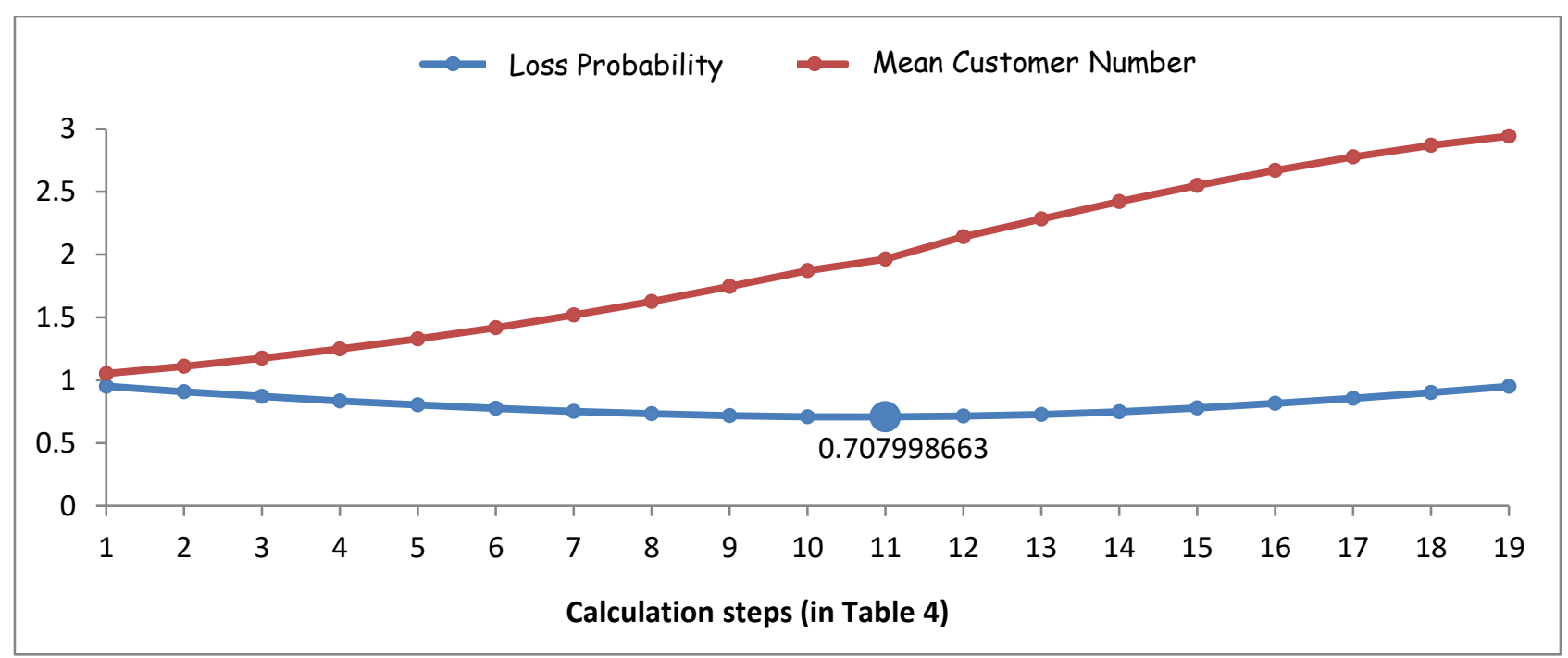

Fig. 2. The obtained $\pi_{\text {loss }}$ and $E(N)$ values of $c=\lambda$ configuration for $\alpha_{1}=\alpha_{2}$

As shown in Figure 2, as in $c=2 \lambda$ configuration, under the condition of $\mu_{1}=\mu_{2}$ and $\alpha_{1}=\alpha_{2}$, the minimum $\pi_{\text {loss }}$ value of $c=\lambda$ configuration is reached, and this value is obtained as $\pi_{\text {loss }}=0.707998663$.

\section{The Simulation of the Model}

In this section, the previously obtained loss probabilities are simulated for $n=100, n=1000$, and $n=$ 10000 using Matlab R2010a program under the system configuration in which they are obtained. As shown in Table 5 and 6 below, previously obtained loss probability $\pi_{l o s s}$ values are found close to the simulation values. This shows that the formula of $\pi_{\text {loss }}$, which is theoretically found with Equation (2.29), is obtained correctly. In Table 7 and 8 , the simulation values are given separately for each of the loss probabilities obtained for $c=\lambda$ and $c=2 \lambda$ configurations, respectively. As seen from these tables, the numerical loss possibilities obtained for each system configuration converge to the simulation results.

Table 5. Comparison of the optimal $\pi_{\text {loss }}$ value obtained for $c=\lambda$ configuration with simulation results

\begin{tabular}{|l|c|c|c|c|c|}
\hline & $\begin{array}{c}\text { Optimal numerical } \\
\text { value }\end{array}$ & $\begin{array}{c}\text { Simulation value } \\
(n=100)\end{array}$ & $\begin{array}{c}\text { Simulation value } \\
(n=1000)\end{array}$ & $\begin{array}{c}\text { Simulation value } \\
(n=10000)\end{array}$ & $\begin{array}{c}\text { Simulation value } \\
(n=1000000)\end{array}$ \\
\hline$\pi_{\text {loss }}$ & 0.707999 & 0.70 & 0.704 & 0.690 & 0.6899 \\
\hline
\end{tabular}


Table 6. Comparison of the optimal $\pi_{\text {loss }}$ value obtained for $c=\lambda$ configuration with simulation results

\begin{tabular}{|l|c|c|c|c|c|}
\hline & $\begin{array}{c}\text { Optimal } \\
\text { numerical value }\end{array}$ & $\begin{array}{c}\text { Simulation value } \\
(n=100)\end{array}$ & $\begin{array}{c}\text { Simulation value } \\
(n=1000)\end{array}$ & $\begin{array}{c}\text { Simulation value } \\
(n=10000)\end{array}$ & $\begin{array}{c}\text { Simulation value } \\
(n=1000000)\end{array}$ \\
\hline$\pi_{\text {loss }}$ & 0.523330711 & 0.53 & 0.519 & 0.521 & 0.5158 \\
\hline
\end{tabular}

Table 7. $\pi_{\text {loss }}$ values and simulation $(n=100000)$ results obtained for $c=\lambda$ configuration

\begin{tabular}{|c|c|c|c|c|}
\hline$\mu_{1}$ & $\mu_{2}$ & $\mu_{3}$ & $\pi_{\text {loss }}($ Numerical $)$ & $\pi_{\text {loss }}$ (Simulation $)$ \\
\hline 0.2 & 0.9 & 0.9 & 0.9091 & 0.9084 \\
\hline 0.9 & 0.55 & 0.55 & 0.7176 & 0.7093 \\
\hline 1.07 & 0.465 & 0.465 & 0.7079 & 0.6899 \\
\hline 1.3 & 0.35 & 0.35 & 0.7279 & 0.6930 \\
\hline 1.8 & 0.1 & 0.1 & 0.9016 & 0.8440 \\
\hline 1.9 & 0.05 & 0.05 & 0.9501 & 0.9107 \\
\hline
\end{tabular}

Table 8. $\pi_{\text {loss }}$ values and simulation $(n=100000)$ results obtained for $c=2 \lambda$ configuration

\begin{tabular}{|c|c|c|c|c|}
\hline$\mu_{1}$ & $\mu_{2}$ & $\mu_{3}$ & $\pi_{\text {loss }}($ Numerical $)$ & $\pi_{\text {loss }}$ (Simulation) \\
\hline 0.2 & 1.9 & 1.9 & 0.9090 & 0.9095 \\
\hline 0.8 & 1.6 & 1.6 & 0.7150 & 0.7192 \\
\hline 1 & 1.5 & 1.5 & 0.6685 & 0.6654 \\
\hline 1.6 & 1.2 & 1.2 & 0.5681 & 0.5667 \\
\hline 1.8 & 1.1 & 1.1 & 0.5468 & 0.5421 \\
\hline 2 & 1 & 1 & 0.5322 & 0.5252 \\
\hline 2.2424 & 0.8889 & 0.8889 & 0.5233 & 0.5158 \\
\hline 2.4 & 0.8 & 0.8 & 0.5267 & 0.5197 \\
\hline 2.6 & 0.7 & 0.7 & 0.5388 & 0.5239 \\
\hline 2.8 & 0.6 & 0.6 & 0.5630 & 0.5387 \\
\hline 3 & 0.5 & 0.5 & 0.6013 & 0.5401 \\
\hline
\end{tabular}

\section{Conclusion and Discussion}

In this study, a blocked stochastic queueing model consisting of parallel service units is given. In this queueing model, the customer arrivals are Poisson distributed with an average of $\lambda$. In the first stage of the system, there is one service unit with an exponential service time with an average of $1 / \mu_{1}$ and in the second stage, there are two service units with exponentially distributed service times, with averages of $1 / \mu_{2}$ and $1 / \mu_{3}$, respectively. This queue model is not allowed to wait in front of the first stage service unit, so there is no queue in this system. An arriving customer is served if the service unit in the first stage is empty. Then, if the service units in the second stage are both empty, the customer continues to the first unit with the probability of $\alpha_{1}$, the second unit with a probability of $\alpha_{2}=1-\alpha_{1}$ or if only one of the service units in the second stage is empty, completes its service in this unit and leaves the system. A third case is that if both service units in the second stage are full, the customer expects at least one of these service units to be empty by blocking the service unit in the 1 st stage. Loss occurs when the customer continues to put into service at the $1^{\text {st }}$ stage service unit or 
when he/she blocks this service unit. One of the main problems in such a queueing model is to calculate this probability of loss and calculate what its optimal value will be. This given stochastic queueing model is mathematically defined by a three-dimensional continuous parameter Markov chain. Limit probabilities of this model are obtained by Kolmogorov difference and differential equations. Afterwards, transition probabilities and blocking probabilities are obtained by the elimination method. The loss probability of the system is found with the help of transition probabilities and blocking probability. In addition, the average number of customers in the system $E(N)$, which is one of the performance measures of the system, is calculated. Since the optimal value of $\pi_{\text {loss }}$ cannot be calculated algebraically, optimal $\pi_{\text {loss }}$ is numerically analysed under the condition $\mu_{1}+\mu_{2}+\mu_{3}=c$ instead. As a result, the optimal $\pi_{\text {loss }}$ value for $c=\lambda$ and $c=2 \lambda$ is reached when $\mu_{2}=$ $\mu_{3}$ and $\alpha_{1}=\alpha_{2}$. The model is simulated with MATLAB R2010 software. The simulation results are compared with the optimal $\pi_{\text {loss }}$ values and the simulation results are found to be very close to the optimal $\pi_{\text {loss }}$ values achieved in the study.

\section{Conflict of Interest}

The authors declare no conflict of interest.

\section{References}

[1] D. Bertsimas, Performance Analysis of Queueing Networks via Robust Optimization, Operations Research 59(2) (2011) 455-466.

[2] K. Jiseung, A. Dudin, S. Dudin, K. Chesoong, Analysis of a Semi-Open Queueing Network with Markovian Arrival Process, Performance Evaluation $120 \quad$ (2018) 1-19. https://doi.org/10.1016/j.peva.2017.12.005

[3] K. Wu, L. McGinnis, Performance Evaluation for General Queueing Networks in Manufacturing System: Characterizing the Trade-off between que and Utilization, European Journal of Operational Research 221(2) (2012) 328-339.

[4] O. Dudina, C. Kim, A. Dudin, S. Dudin, Retrial Queuing System with Markovian Arrival Flow and Phase-Type Service Time Distribution, Computers \& Industrial Engineering 66(2) (2013) 360-373.

[5] G. C. Hunt, Sequential Arrays of Waiting Lines, Operations Research 4(6) (1956) 674-683.

[6] S. Kolledath, K. Kumar, Performance Analysis of Series Queue with Customer's Blocking, Performance Prediction and Analytics of Fuzzy, Reliability and Queuing Models (2020) 191-201.

[7] G. Basharin, V. Naumov, K. Samouylov, On Markovian Modelling of Arrival Processes, Stat Papers 59 (2018) 1533-1540. https://doi.org/10.1007/s00362-018-1042-9

[8] I. F. Akyıldız, H. V. Brand, Exact Solutions for Networks of Queues with Blocking-After-Service, Theoretical Computer Science 125(1) (1994) 111-130.

[9] M. Zobu, V. Sağlam, M. Sağır, E. Yücesoy, T. Zaman, The Simulation and Minimization of Loss Probability in the Tandem Queueing with Two Heterogeneous Channels, Mathematical Problems in Engineering Article ID 529010 (2013) 4 pages. http://dx.doi.org/10.1155/2013/529010

[10] V. Sağlam, M. Zobu, A Two-Stage Model Queueing with No Waiting Line between Channels, Mathematical Problems in Engineering Article $\quad$ ID $679369 \quad$ (2013) 5 pages. http://dx.doi.org/10.1155/2013/679369 